BLS 32, No 1 2006. DOI: http://dx.doi.org/10.3765/bls.v32i1.3451 (published by the Berkeley Linguistics Society and the Linguistic Society of America)

\title{
Re-binding and the Derivation of Parallelism Domains
}

\author{
DANIEL HARDT \\ Copenhagen Business School
}

\section{The Re-binding Puzzle}

How can it be that meaning arises in the absence of overt linguistic structure? This is the puzzle of ellipsis - a puzzle that has been a major preoccupation in theoretical linguistics for several decades. One intriguing aspect of the puzzle concerns the phenomenon of sloppy identity, illustrated with VP ellipsis (VPE) in (1):

(1) John said Mary hit him. Bill did too. (said Mary hit John/Bill)

Here, the antecedent VP is said Mary hit him, with him referring to John. The elided VP is ambiguous between a strict reading, Bill said Mary hit John and a sloppy reading, Bill said Mary hit Bill. Surprisingly, this ambiguity seems to disappear in the following variant of (1):

(2) John said Mary hit him. Bill said she did too. (hit John/*Bill)

Here, there emerges a clear preference for the strict reading hit John. What might account for this remarkable fact? In this paper, I will suggest that this rebinding puzzle reflects a fundamental fact about the computational system which links syntactic structure with meaning. I will propose that this system operates in a monotonic fashion: that is, meaning representations are constructed as early as possible during a bottom-up derivation, and the resulting meaning representations cannot be revised later.

In what follows, I begin with the original account of the re-binding puzzle, due to Sag (1976), who first observed the phenomenon. I argue that Sag's account is unsuccessful, and I continue with a proposed Re-binding Generalization: namely that re-binding is possible only when necessary to satisfy parallelism. I next show that this generalization follows if the syntax-semantics interface operates monotonically. At this point, I describe a survey I performed which supports Sag's original observations concerning re-binding - this is important, because the observations are subtle, and have been questioned in the literature. Next, I turn to a puzzle concerning focused pronouns. I argue that the Monotonic Derivation system makes available a 
simpler, and more empirically successful account than has previously been available. Finally, I compare the proposed account with an alternative account, based on a constraint called MaxElide.

\subsection{Sag's Explanation: No Re-binding}

In Sag's account of VP ellipsis, the VP is represented as a lambda abstract, so that the elided VP in (1) is: $\lambda \mathrm{x}$. $\mathrm{x}$ said Mary hit $\mathrm{x}$. On this representation, this pronoun is represented as a lambda-bound variable, giving rise to the sloppy reading. Note that the sloppy pronoun is bound within the elided VP. The sloppy pronoun in (2) must be bound outside the elided VP.

Following Takahashi and Fox (2005), I will use the term re-binding to describe cases where a pronoun is bound outside the elided material. According to Sag, rebinding is not permitted. Any variables in the logical representation of the elided VP must be bound within it.

\subsection{But-Re-binding IS Possible}

Sag's explanation, while admirably simple, cannot be maintained. It has become widely accepted that re-binding is possible under certain conditions, as illustrated by (3):

(3) Nearly EVERY boy said Mary $_{2}$ hit him ${ }_{1}$. But BILL 3 didn't say she did. (hit him 3 )

Here, the sloppy pronoun $\operatorname{him}_{3}$ is bound outside the elided VP, but the sloppy reading is acceptable. This shows that an alternative generalization is needed concerning re-binding.

\section{The Re-binding Generalization}

We have now seen three examples: for (1), there is no re-binding, since the pronoun is bound within the elided VP. Here strict and sloppy are thus equally possible. For (2) the sloppy reading requires re-binding - it would require the pronoun to be bound outside the VP. Since the strict reading is available to satisfy parallelism, the sloppy reading is blocked. Finally, with (3), the strict reading is not available, since the pronoun in the first sentence is bound by the quantified NP Nearly every boy. The only possibility is the sloppy reading, which is therefore permitted even though it involves re-binding. Based on these observations, I propose the following generalization:

Re-binding Generalization: Re-binding is possible only when necessary to satisfy parallelism.

\subsection{Additional Evidence}

In the following example, re-binding is not possible, as observed by Bach and Partee (1980): 
(4) Bill ${ }_{1}$ BELIEVES that Sally 2 will marry him , but everyone $_{3}$ KNOWS that she 2 WON'T. (marry him 1 /* him 3 )

Here, the strict reading is available, thus blocking the sloppy re-binding reading. In the following variant of (4), the strict reading is made unavailable, and the rebinding reading emerges as acceptable.

(5) Everyone ${ }_{1}$ HOPES that Sally 2 will marry him , but Bill $_{3}$ KNOWS that she will. (marry *him $1 /$ him $_{3}$ )

This supports the proposed re-binding generalization.

\section{Proposal: Monotonic Derivation of Parallelism Domains}

I propose that the re-binding generalization reflects a monotonicity constraint on the derivation of semantic representations, as follows:

Monotonic Derivations: As soon as a Parallelism Domain can be identified during a bottom-up derivation, indexation takes place, and cannot be modified later in the derivation.

My claim is that the relevant effects all follow from this monotonicity property. To see this, it is necessary to first clarify several related notions. We begin with two definitions:

- Parallelism Domain (PD): A constituent E is a Parallelism Domain if there is an antecedent $\mathrm{A}$ such that there is a valid indexing $\mathrm{E}^{\prime}$ of $\mathrm{E}$ such that $\mathrm{E}^{\prime}$ is Parallel to A.

- Valid indexing: a pronoun (or other variable) must have an index $i$, such that $\operatorname{i\varepsilon Dom}(F)$, where $F$ is the File representing the current state of the discourse. (Familiarity (Heim (1982))/Accessibility (Kamp and Reyle (1993)))

I now give an account of parallelism.

\subsection{Determining Parallelism}

To determine if two constituents $\mathrm{A}$ and $\mathrm{B}$ are parallel, lambda-abstract over Parallel Elements, $\mathrm{P}_{A}$ and $\mathrm{P}_{B}$, giving $\left[\mathrm{P}_{A}, \lambda\right.$ x. $\left.\mathrm{A}^{\prime}\right],\left[\mathrm{P}_{B}, \lambda\right.$ y. $\left.\mathrm{B}^{\prime}\right]$. If $\mathrm{A}^{\prime}$ is identical to $\mathrm{B}^{\prime}$, Parallelism is satisfied.

It is standard to permit two "exceptions" to the identity condition: focused elements need not be identical, and lambda-bound variable indices need not be identical ("alphabetic variance" condition). Finally, the lambda abstraction step works as follows: substitute lambda-bound variable $x$ for $\mathrm{P}_{A}$ in A. If A contains an element $p$ coindexed with $\mathrm{P}_{A}, \mathrm{x}$ may also be substituted for $p$ (Dalrymple et al. (1991)). Thus lambda abstraction permits an option with respect to coindexed pronouns-they are optionally replaced with a lambda-bound variable.

We illustrate with example (1). Here, constituents A and B are as follows: 
$\mathrm{A}=J_{o h n_{1}}$ said Mary 2 hit him $1 \quad \mathrm{~B}=$ BILL 3 did too. (said Mary 2 hit him 3 )

We determine parallel elements and perform lambda-abstraction:

Parallel Elements: John, Bill

Lambda-Abstract:

John $_{1}, \lambda$ x. $x$ said Mary 2 hit $x \quad$ BILL $_{3}, \lambda$ y. $y$ said Mary 2 hit $y$

We can see that parallelism is satisfied: John and BILL are non-identical, but BILL is focused. Also there are different variables, but this is permitted by the alphabetic variance condition.

\subsection{Illustrating the Proposal}

We now see how the re-binding generalization follows from the Monotonic Derivations proposal. We begin with (2), where re-binding is not permitted.

(2) $\mathrm{John}_{1}$ said Mary 2 hit him 1 . Bill said she did too. (hit him)

Here, the smallest potential PD is [did hit him]. This is indeed a PD, with valid indexing [did hit him 1 ]. The strict reading results, and re-binding is not possible.

We turn now to (3), where rebinding is permitted:

Nearly every boy ${ }_{1}$ said Mary 2 hit him 1 .

But Bill 3 didn't say she did (hit him)

$\cdots$

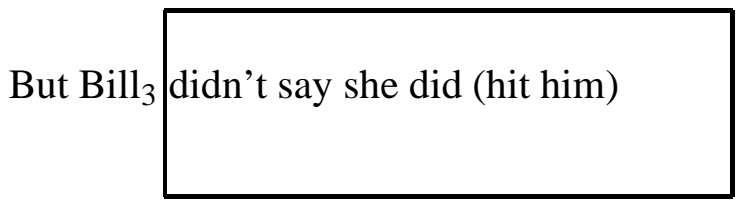

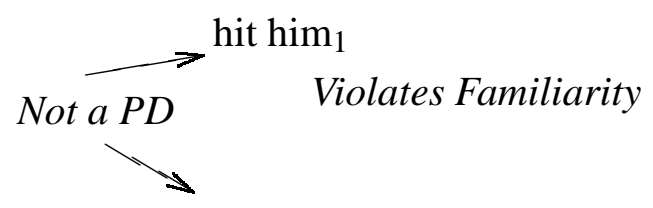

hit him 3 Not Parallel

Is a PD

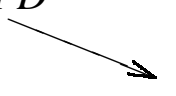

y didn't say she 2 did hit y

We begin again with the smallest possible PD [did hit him]. In this case, there is no valid indexing which allows it to be a PD: him 1 violates Familiarity, since the index 1 is associated with the binder every boy $y_{1}$, which only had scope over the previous sentence. The index 3 is available, but this causes parallelism to fail. The first stage at which parallelism is satisfied is where the maxtrix VP has been constructed. At this point it is clear that parallelism is satisfied (we ignore the negation for simplicity):

Nearly EVERY boy $1,[\lambda$ x.x said Mary 2 hit $x]$
BILL $_{3}$,
$[\lambda$ y.y said Mary 2 hit $y]$

Here, we derive the sloppy reading. 


\section{Re-binding and the Derivation of Parallelism Domains}

\subsection{Pragmatics and Strict/Sloppy Blocking}

We have seen that re-binding becomes possible when the strict reading is structurally ruled out (because of quantifier scope), as in (3). The strict reading can also be ruled out by factors that are not purely structural, such as pragmatic preferences. This is supported by the following example:

(6) MARY might admit that the criminals had been in contact with her, but SUSAN wouldn't admit that they had (been in contact with $\mathrm{Su}$ san/?Mary).

Here, the strict reading is not structurally ruled out, since the binder is a name (Mary), not a quantifier. Instead, the strict reading is problematic for pragmatic readings, more specifically the verb admit suggests that if A admits $\mathrm{P}, \mathrm{P}$ is reflects negatively on A. Thus the strict reading is pragmatically degraded—why should it be an admission for Susan that the criminals had been in contact with Mary?

This pragmatic factor allows the derivation to continue to the point at which the rebinding reading is established. Example (6) was included in a recent survey, which supported the judgment that the re-binding reading is preferred. I now turn to the results of this survey.

\section{What are the Facts?}

The contrast between (1) and (2), first observed by Sag (1976) was the point of departure for this paper. This judgment, if correct, contradicts many recent accounts, such as Dalrymple et al. (1991), Fiengo and May (1994), Hardt (1999). However, the judgment has been called into question by many theorists (Hardt (1993), Asher (1993)).

To shed some light on this, I performed a survey to test re-binding judgments, using Linguist-GRID — a web-based tool for interactive linguistic surveys, developed by Matthias Kromann at Copenhagen Business School. The survey was advertised on the LINGUIST List and elsewhere in Nov/Dec 2004. It involved 29 subjects, who rated 30 examples of VP ellipsis. Each example was presented together with the reading to be evaluated (parenthesized in italics). Examples were rated on the following Four-point scale: [0] Fully Acceptable [1] Closer to Acceptable [2] Closer to Unacceptable [3] Fully Unacceptable.

Overall, the results support Sag's claim that there is a difference between (1) and (2). But they also show that the "bad" rebinding examples receive a mildly degraded status, rather than fully unacceptable. 
Daniel Hardt

\subsection{Linguist-GRID Survey Results}

\begin{tabular}{|l|c|}
\hline Example Type & Mean Judgment \\
\hline Strict & .66 \\
John said Mary hit him. & \\
Harry did too. (said Mary hit John) & \\
\hline $\begin{array}{l}\text { Sloppy (no re-binding) } \\
\text { John said Mary hit him. }\end{array}$ & .61 \\
Harry did too. (said Mary hit Harry) & \\
\hline $\begin{array}{l}\text { Re-binding } \\
\text { John said Mary hit him. }\end{array}$ & 1.16 \\
Harry said she did too. (hit Harry) & \\
\hline $\begin{array}{l}\text { Re-binding (Strict Blocked) } \\
\text { Nearly EVERY boy said Mary hit him. } \\
\text { But BILL didn't say she did. (hit Bill) }\end{array}$ & \\
\hline
\end{tabular}

As might be expected, strict and sloppy (no re-binding) receive similar scores of around .6. The third category receives a somewhat degraded status, consistent with Sag's observation that re-binding in such cases is degraded, although not fully unacceptable, as Sag had originally claimed. The fourth category contradicts Sag's claim, and supports the proposed approach: here the strict reading is unavailable, and the sloppy re-binding reading is even more acceptable on average than the nonre-binding categories.

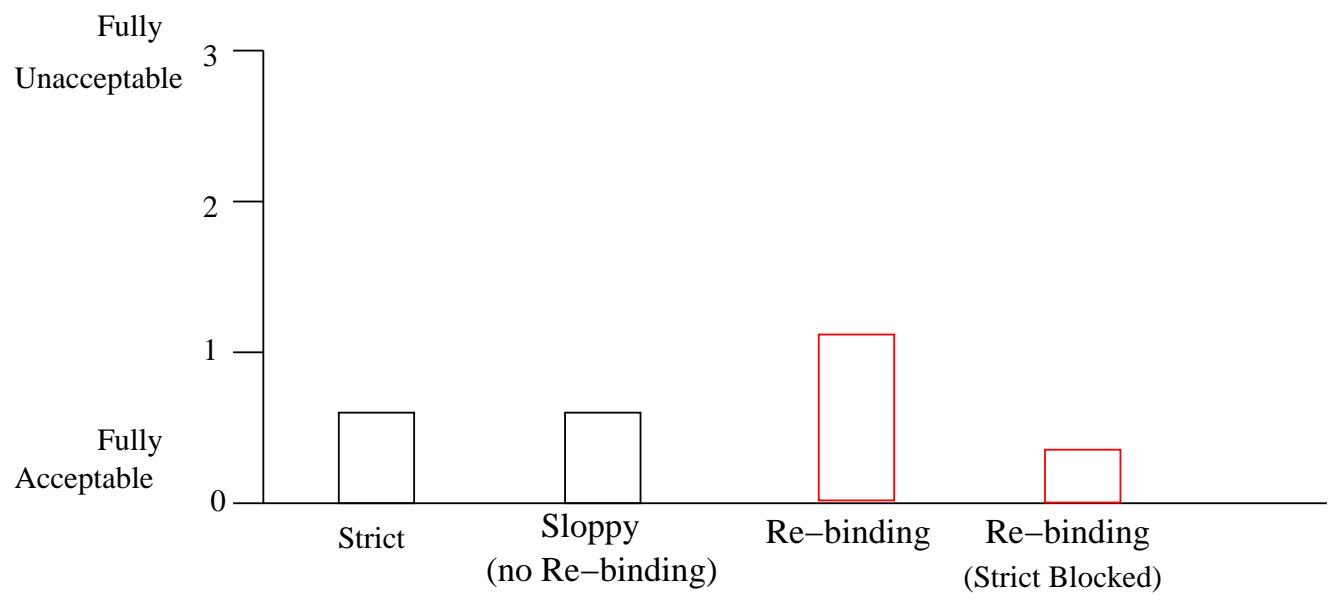

Complete results of the survey can be found on the Linguist-GRID.org web site.

\section{Pronouns and Focus}

I turn now to a puzzle concerning focus and bound pronouns, discussed in recent papers by Sauerland (to appear) and Jacobson (2000). Consider (7): 


\section{Re-binding and the Derivation of Parallelism Domains}

Every boy likes his father, and every TEACHER likes HIS father.

Focus is possible on HIS if it is interpreted as bound by every TEACHER. As pointed out by both Sauerland and Jacobson, this is surprising, in view of the widespread assumption that a focused expression must contrast with some other expression. Sauerland and Jacobson propose that these pronouns do contrast, in terms of what might be called Hidden Content: for Sauerland, bound pronouns contain a silent property, while for Jacobson they are associated with contrasting domains.

Both Sauerland and Jacobson consider and reject an explanation in terms of $\mathrm{Fo}^{-}$ cus Agreement: a bound pronoun can be focused if its binder is focused. However, I will show that the apparent problems with Focus Agreement disappear when the Monotonic Derivations approach is adopted. Furthermore, I will point out problems that arise for the Hidden Content proposal, which do not arise for the Focus Agreement approach. The Monotonic Derivations account plays a key role in this argument; thus, to the extent that this argument is successful, it provides additional support for the Monotonic Derivations proposal.

The proposed Agreement Condition can be stated as follows:

Agreement Condition: Given a PD $A$ containing a pronoun $x$ with a binder $B$, focus on $x$ is licensed if focus on $B$ is licensed.

Furthermore, I will follow Sauerland and Jacobson in assuming the following Contrast Condition: given a PD A containing a focused constituent $\alpha$, we require

that $A$ would not be a PD without focus on $\alpha$ (Sauerland (to appear), Schwarzschild (1999)).

Here is Sauerland's representation of (7):

Every boy likes [the BOY's] father, and every TEACHER likes [the TEACHER's] father.

The bound pronouns his receive hidden content from their binders-the nominals boy and teacher. Now, contrast between TEACHER and BOY is licensed in the normal way.

\subsection{The Apparent Problem with Focus Agreement}

Sauerland and Jacobson consider and reject the Focus Agreement account, because of examples like (9):

(9) Every BOY called his $_{1}$ mother before every TEAcher 2 called $* \mathrm{HIS}_{1}$ mother. (Sauerland (to appear))

As Sauerland points out, focus on the pronoun $H I S_{1}$ is impossible, despite the fact that it has a focused binder (Every $\mathrm{BOY}_{1}$ ). However, on the proposed Monotonic Derivations account the index ${ }_{1}$ is not permitted on focused HIS. To see this, we examine the derivation, under the assumption that HIS is focused. We begin with 
[HIS 1 mother]: this fails to be a PD, because it violates the Contrast Condition-it would be a PD without focus on his. The derivation continues until we construct [every TEAcher 2 called HIS mother] - this is a PD, with the indexing $\mathrm{HIS}_{2}$. At this point, HIS $_{1}$ would again violate the Contrast Condition. Since a PD is identified at this point, this is the only indexing permitted.

As both Sauerland and Jacobson acknowledge, the Agreement Condition is the simplest account of these facts. With Monotonic Derivations, the apparent problem with Focus Agreement is shown to be illusory. Next, I show that the Hidden Content account encounters serious empirical problems.

\subsection{What is the Hidden Content?}

One possible view, which I will call the Conservative Hidden Content View, is that the Hidden Content is the nominal restrictor of the binder. The Liberal Hidden Content View, which both Sauerland and Jacobson appear to end up with, is that Hidden Content can be any property presupposed to be true of the relevant individual. Regardless of which view one adopts, the Hidden Content view encounters important empirical problems involving strict and sloppy pronouns.

The following examples illustrate that focus is never permitted when a pronoun receives a strict reading.

(10) John 1 likes his 1 father, and BILL 2 likes his $1 / * \mathrm{HIS}_{1} / \mathrm{HIS}_{2}$ father too.

(11) A man likes his $_{1}$ father, and ANOTHER man 2 likes his ${ }_{1} / * \mathrm{HIS}_{1} / \mathrm{HIS}_{2}$ father too.

(12) THIS $\operatorname{man}_{1}$ likes his ${ }_{1}$ father, and THIS $\operatorname{man}_{2}$ likes his $1 / * \mathrm{HIS}_{1} / \mathrm{HIS}_{2}$ father too.

In (10) and (11), if the second his is focused, it cannot be interpreted strictly. Let's look at (11): we start with [likes HIS father]. This is not a PD-[likes HIS father] violates Contrast Condition (ie., focus wasn't needed), and [likes $\mathrm{HIS}_{2} \mathrm{fa}$ ther] violates Familiarity (index 2 not yet available). We continue until we construct [ANOTHER man $_{2}$ likes HIS father]. With HIS ${ }_{1}$, this is not a PD - it violates the Contrast Condition. But [ANOTHER man 2 likes HIS 2 father] is a PD, with focus on HIS licensed by Agreement.

All the facts in (10)-(12) are captured by the Focus Agreement/Monotonic Derivations proposal. As we will see, these facts are problematic for the Hidden Content view.

\subsection{Hidden Content: Problems with Strict and Sloppy Pronouns}

Can the facts in (10)-(12) be accounted for with Hidden Content? Let's start with the Conservative View-in this case, we can argue that focus is not permitted for the strict reading in examples like (10), since the strict pronoun would presumably inherit the same restrictor as the antecedent pronoun. But this is far too restrictivewe are left with no means to tell the strict and sloppy readings apart for examples 


\section{Re-binding and the Derivation of Parallelism Domains}

like (11) and (12). In (12) the two antecedent have exactly the same lexical material ("this man").

What about the Liberal View? Here, we allow the hidden content to be any presupposed property. But then nothing ensures, for example (10), that the strict pronoun has the same hidden content as the antecedent pronoun. To illustrate this, consider a context in which it is known that John is both a lawyer and a doctor. Then we could have a strict reading where the representation is:

$$
\mathrm{John}_{1} \text { likes [the }{ }_{1} \text { doctor's] father, and BILL } 2 \text { likes [the }{ }_{1} \text { lawyer's] father. }
$$

For the strict reading, the Hidden Content account would incorrectly permit focus here. Finally, consider (11), where the two men are completely indistinguishable. The sloppy reading still permits stress-but there is no contrastive Hidden Content, so stress is incorrectly ruled out on the Hidden Content view.

These problems simply don't arise for the Focus Agreement/Monotonic Derivations approach, which simply permits focus agreement for bound pronouns. This account crucially relies on the Monotonic Derivations approach.

\section{Comparison with MaxElide}

To my knowledge there is only one other account that attempts to capture Sag's observations concerning re-binding: this is the account of Takahashi and Fox (2005), based on a condition termed MaxElide, originally due to Merchant (to appear). After presenting the MaxElide account, I will argue that the Monotonic Derivations proposal is simpler and appears to be more successful empirically. However, there are additional differences between the two proposals, which deserve further investigation.

\subsection{The Account}

The Maxlide account consists of the following requirement on ellipsis: Elide the biggest elidable constituent reflexively dominated by $P D$. In other words, ellipsis must be maximal within some Parallelism Domain. To see if a given elided constituent $\mathrm{C}$ satisfies MaxElide, one must find the smallest PD containing C. If PD contains an elidable constituent $\mathrm{C}^{\prime}$ that contains $\mathrm{C}$, the ellipsis violates MaxElide. This means that if $\mathrm{C}$ contains a sloppy pronoun $p$, the minimal PD must contain the binder for $p$. Furthermore, for strict readings, MaxElide is always trivially satisfied, because elided constituent $\mathrm{C}$ itself is always a PD.

MaxElide correctly rules out the sloppy reading for (2), repeated below:

$$
\text { John }_{1} \text { said Mary } 2 \text { hit him } 1 \text {. Bill said she did too. (hit him) }
$$

Here, the smallest containing PD is [Bill said she did too. (hit him)]. But this PD contains a larger constituent—-the containing VP said she hit him, that could have been elided. Thus the sloppy reading for (2) violates MaxElide. 
Daniel Hardt

However, MaxElide does not capture the other main effect discussed in this paper: when strict is blocked, the re-binding reading is acceptable, as in (3), repeated here:

Nearly EVERY boy 1 said Mary 2 hit him 1 . But BILL 3 didn't say she did. (hit him 3 )

Here, the sloppy reading is incorrectly ruled out by MaxElide, since a larger ellipsis was possible within the smallest PD containing ellipsis-namely the entire sentence. Furthermore, MaxElide incorrectly permits a sloppy reading in (4), repeated below:

Bill $_{1}$ BELIEVES that Sally 2 will marry him ${ }_{1}$, but everyone 3 KNOWS that she 2 WON'T.

This is because the Intervening Focus on WON'T makes it impossible to elide anything bigger.

\subsection{Derivational Perspective}

The MaxElide constraint makes no reference to derivations; however, in a footnote Takahashi and Fox consider a derivational formulation:

One attractive implementation of our idea relies on the assumption that deletion can apply at the course of the derivation... In the Re-binding context, deletion cannot apply until a re-binder is introduced into the derivation, since the parallelism condition is not met before that stage of the derivation. (Takahashi and Fox 2005, fn. 7)

In the Monotonic Derivations approach, it is indexation (rather than deletion) which applies derivationally. Furthermore, once this derivational perspective is taken, the relevant facts are captured without any appeal to MaxElide. Indeed, MaxElide fails to capture the strict/sloppy blocking effects. Thus the Monotonic Derivations account would appear to be both simpler and more empirically successful. However, there are other differences between the two proposals which require further study. I consider two of these below: intervening focus, and large vs. small ellipsis.

\subsection{Intervening Focus}

MaxElide requires ellipsis of the biggest elidable constituent dominated by PD. A consituent contain a focused element is not elidable; thus Intervening Focus can allow a smaller ellipsis that would otherwise not be permitted.

$\mathrm{John}_{1}$ argued that Mary hit him 1 , but BILL 2 DENIED that she did. (hit $\operatorname{him}_{2}$ ) 


\section{Re-binding and the Derivation of Parallelism Domains}

Here, Takahashi and Fox argue that the sloppy reading is acceptable, because of the Intervening Focus, DENIED. This is what is predicted by MaxElide. The Monotonic Derivations proposal does not permit the sloppy reading. To my ear, the sloppy reading remains degraded here, just as it is without the Intervening Focus. This is supported by results from the LinguistGRID survey described above, where sentences of the form of (17) did not have acceptable sloppy readings.

\subsection{Large vs. Small Ellipsis}

MaxElide predicts that ellipsis of constituent $\mathrm{C}$ will in general block ellipsis of a contained constituent $\mathrm{C}^{\prime}$, if the smallest PD contains $\mathrm{C}$. This applies to cases in which the binding relationship involves a wh-operator, as seen in the contrast between (18)a and (18)b.

(18) a. John knows which professor we invited, but he is not allowed to reveal which $_{x}$ one. (we invited $x$ )

b. *John knows which professor we invited, but he is not allowed to reveal which ${ }_{x}$ one we did. (invited $x$ )

Here, the smallest PD contains the sluiced IP (we invited $\mathrm{x}$ ) as well as the contained VP (invited $\mathrm{x}$ ), since the binder for $x$ is which $_{x}$. (18)b is ruled out by MaxElide since a larger ellipsis is possible within this PD.

The Monotonic Derivations proposal does not capture this contrast; it permits the re-binding reading in both (18)a and (18)b. However, there is reason to doubt the MaxElide account of this contrast. Consider the following observation:

(19) John won't say who we should hire, but

(20) a. Harry will. (say who $x$ we should hire $x$ )

b. *Harry will say who . $^{*}$ (we should hire $x$ )

While (20)b is degraded just as (18)b is, MaxElide doesn't rule out (20)b. Here, the CP "who ${ }_{x}$ we should hire $\mathrm{x}$ " is a PD, and the sluice in (20)b is maximal within that PD. There are clearly interesting contrasts involving the large vs. small ellipsis in wh-binding cases. However, it is not clear if these can be captured by an account based on MaxElide.

\section{Final Thoughts}

The Monotonic Derivations proposal has much the same structure as the syntaxphonology mapping termed Cyclic Linearization, which Fox and Pesetsky (2005) describe as follows:

... structure is built from "bottom to top"... mapping between syntax and phonology (Spell-out) takes place at various points in the course of the derivation... information about linearization, once established at the end of a given Spell-out domain, is never deleted in the course of a derivation. 
Daniel Hardt

In a similar way, information about indexation, once established during a derivation, is never changed later. This suggests the possibility of an attractive unification, in which syntax interfaces with both sound and meaning in a simple, monotonic fashion.

Intuitively, monotonicity is an attractive property for a derivation system, at least in the sense that it would appear to simplify the computation required. I have argued that monotonicity in the construction of semantic representations explains several observations concerning re-binding that have not been previously accounted for, and it also accounts for certain puzzles involving focused pronouns. However, many of the key judgments are subtle and controversial. While the informal survey reported above may have clarified this situation, there is no doubt that more systematic and far-reaching empirical investigation is required.

\section{References}

Asher, Nicholas. 1993. Reference to Abstract Objects in English. Dordrecht: Kluwer.

Bach, E., and B. Partee. 1980. Anaphora and Semantic Structure. In A. Ojeda, ed., Papers from the Parasession on Pronouns and Anaphora, 1-28. Chicago: Chicago Linguistic Society.

Dalrymple, Mary, Stuart Shieber, and Fernando Pereira. 1991. Ellipsis and HigherOrder Unification. Linguistics and Philosophy 14(4).

Fiengo, Robert, and Robert May. 1994. Indices and Identity. Cambridge, MA: MIT.

Fox, Danny, and David Pesetsky. 2005. Cyclic Linearization of Syntactic Structure. Theoretical Linguistics 31(1-2).

Hardt, Daniel. 1993. Verb Phrase Ellipsis: Form, Meaning, and Processing. Ph.D. diss., University of Pennsylvania.

Hardt, Daniel. 1999. Dynamic Interpretation of Verb Phrase Ellipsis. Linguistics and Philosophy 22(2):187-221.

Heim, Irene. 1982. The Semantics of Definite and Indefinite Noun Phrases, Ph.D. diss., University of Massachusetts, Amherst.

Jacobson, Pauline. 2000. Paychecks, Stress, and Variable-Free Semantics. In Proceedings of Semantics and Linguistic Theory XI.

Kamp, Hans, and Uwe Reyle. 1993. From Discourse to Logic. Dordrecht: Kluwer.

Merchant, Jason. To appear. Variable Island Repair under Ellipsis. In Kyle Johnson, ed., Topics in Ellipsis. Oxford: Oxford University Press.

Sag, Ivan A. 1976. Deletion and Logical Form, Ph.D. diss., Massachusetts Institute of Technology.

Sauerland, Uli. To appear. The Content of Pronouns: Evidence from Focus. In Kyle Johnson, ed., Topics in Ellipsis. Oxford: Oxford University Press.

Schwarzschild, Roger. 1999. GIVENness, AvoidF and other Constraints on the Placement of Focus. Natural Language Semantics 7(2):141-177. 


\section{Re-binding and the Derivation of Parallelism Domains}

Takahashi, Shoichi, and Danny Fox. 2005. MaxElide and the Re-binding Problem. In Proceedings of Semantics and Linguistic Theory XV. Los Angeles, CA.

Daniel Hardt

Copenhagen Business School

Department of Computational Linguistics

Denmark

dh.isv@cbs.dk 